\title{
STATISTICAL PROPERTIES OF THE YIELD STRENGTH OF NORMAL STRENGTH HULL STRUCTURAL STEEL PLATES
}

\author{
Zbigniew Sekulski \\ West Pomeranian University of Technology, Szczecin \\ Faculty of Maritime Technology and Transport, \\ al. Piastów 41, 71-065 Szczecin, Poland \\ E-mail: zbych@zut.edu.pl
}

\begin{abstract}
The statistical properties were studied of normal strength hull structural steel plates (with a strength level of 235) of a thickness of $\leq 50 \mathrm{~mm}$ that were manufactured in 1990-1991 at four European steelworks. The study focused on the yield strength, as the available literary sources indicate that in terms of structural strength and design assessment this particular aspect is the most essential property of steel used for ship structures, and, at the same time, its values are characterized by the highest variability. Detailed statistical tests were performed on nearly 2,200 plates. The results of these analyses suggested that for the sample examined herein the mean yield strength value was $308.3 \mathrm{MPa}$, the standard deviation was $25.24 \mathrm{MPa}$, the coefficient of variation was 0.0819 (8.19\%), the (average) difference bias was $73.3 \mathrm{MPa}$ and the (average) ratio bias was 1.31. It has been shown that both lognormal distribution $L N(5.7276,0.0817)$ and normal distribution $N(308.2589,25.2444)$ provide an adequate representation of normal strength hull structural steel plates.
\end{abstract}

Keywords: Normal strength hull structural steel, plates, yield strength, strength uncertainty, statistical analysis, statistical properties, probability density function, Kolmogorov-Smirnov goodness-of-fit test

\section{INTRODUCTION}

The key to the application of computational methods based on the probability theory is to have reliable information on the statistical properties of the phenomena. In the case of ship structures, this first of all applies to loads and the physical and geometrical features of the structure.

From the point of view of the rules adopted by classification societies, the physical characteristics of a structure are its yield strength, tensile strength, Young's modulus, Poisson's ratio, the relative elongation of a specimen after fracture, and the impact energy. Some of them, such as the yield strength, the tensile strength, the relative elongation of a specimen after fracture and the impact energy, are tested by the manufacturer and confirmed with a classification society certificate. For these characteristics, probability density mathematical models can be formulated on the basis of data recorded in the relevant certificates of classification societies. The other ones, such as the Young's modulus or the Poisson's ratio, are not confirmed by classification society certificates. Among the materials' physical characteristics determined in laboratory tests and confirmed by certificates, the yield strength is the only one that explicitly appears in the calculation formulas for scantling calculations of structural components: plate thickness, section modulus, the cross sectional area and the moment of inertia of 
stiffeners and frames. Therefore, it can be suggested that in terms of the structural reliability analysis and the rule-based structural design, the yield strength is the key physical characteristic of ship hull structures. In this work, the focus is only on the yield strength of normal strength hull structural steel.

The objective of this investigation is to find the probabilistic characteristics of the yield strength to be used in the reliability analysis and design of ship structures. This search for random variables mainly in terms of their means, standard deviations, COVs and probability distributions can be achieved in two steps: (1) by collecting data and (2) by analyzing such data. The first step is to collect as many samples of data deemed to be appropriate for representing the random variables under investigation as possible. The second one is to statistically analyze the collected data in order to determine the probabilistic characteristics of these variables. As Hess et al. (2002) note that "The impact of the random uncertainty on structural strength prediction cannot be overemphasized, and it is a very important issue in reliability-based analysis of" ship structures. The objective of the investigation is therefore to present statistical estimates of the uncertainty associated with the yield strength of hull structural steel plates used in the analysis and design of ship structures.

Colette (2017) wrote that "As objects built in either bespoke or short-run production settings, ship structures are primarily assembled from standardized structural components. Hence, statistical data on underlying material and structural shape properties are critical sources of variability." Simiu and Smith (1984) stated that "Comprehensive probabilistic descriptions of the relevant physical properties of the structure are frequently not available." Although a long time has elapsed since this statement was made, it is still valid and, consequently, reliable data continue to be in short supply.

The objective of this paper was limited to a statistical investigation of the yield strength of normal strength hull steel plates. The data on the yield strength came from casts made in 1990-1991 at three Polish and one Danish steelworks. Years later, such data could still be of use for assessing ship strength, as the author is unfamiliar with any papers showing the correlation between strength properties and time. Therefore, the results presented here may also today constitute a valuable empirical basis for theoretical research or numerical calculations.

\section{METHODOLOGY}

The main goal of the paper was to study the bias between the actual (measured) value of the yield strength (YS) $R_{e}$ and the values (rule-required) used for design, and also to create a statistical (probabilistic) model of this bias for use in reliability analysis and design methods.

The paper provides detailed statistical data for the YS of steel plates used in ship structures. These data were collected from four manufacturers with the objective of identifying strength uncertainties. The resultant data included the mean $(\mu)$, the standard deviation $(\sigma)$, the coefficient of variation $(C O V)$, and the probability distribution model for yield strength. Also included were other types of data that could be of interest to and use for the readers, such as the median, and others.

The plates that the data were based on were made of normal strength hull structural steel (NSHSS), with a minimum value of yield strength (rule-required) $R_{e, r u l e}:=R_{e}=$ $235 \mathrm{MPa}$ (a strength level of 235) and an ultimate strength $R_{m}=400-520 \mathrm{MPa}$ (the nomenclature and values are as per IACS 2017).

The investigation used data on the thickness and yield strength of NSHSS plates manufactured in 1990 and 1991 at three Polish and one Danish steelworks.

The plates had been certified by the Polish Register of Shipping classification society in accordance with the IACS requirements. The plate thicknesses provided in the 
paper were nominal values as stated in the orders, while the YS values were actual values obtained through measurements made by the manufacturer and stated in the classification certificates. The nominal thicknesses of the plates fell within the range from 1 to 50 $\mathrm{mm}$.

The material strength variable considered in this paper is the YS. As Hess et al. (2002) stated, "a means of addressing the uncertainty inherent in structural variables is to study the bias between the actual (measured) value and the value used for design and to create a statistical (probabilistic) model of this bias for use in reliability analysis and design methods". Bias is a random discrepancy between a nominal value and a true value of YS. According to Hess et al. (1997), the uncertainty in the YS is quantified using two types of bias, the ratio bias and the difference bias. The ratio bias is the ratio between the measured value and the nominal (or design) value for YS as follows: $b_{R}=$ (measured value)/(nominal value). ${ }^{1}$ The difference bias is the difference, or error, between the measured value and the nominal value: $b_{D}=$ (measured value) - (nominal value).

Statistical information on the YS is summarized in the following section. The yield strength ratio was computed as the mean to nominal value of the YS. The section also provides information such as the number of plates, the arithmetic mean, the median, the standard deviation and the coefficient of variation $(C O V)$, as well as the probability distribution types and their parameters.

The paper presents three theoretical probability density functions (PDF) for YS distribution, namely the normal distribution, the lognormal distribution and the Weibull distribution. The Dell ${ }^{\mathrm{TM}}$ Statistica $^{\mathrm{TM}} 13.1$ software was used to explore which of the

\footnotetext{
${ }^{1}$ The excess ratio bias may be used, as well. The excess ratio bias is defined as the ratio bias minus 1.0.
}

PDFs were the most representative of the sample data. The PDFs were ranked using Kolmogorov-Smirnov goodness-of-fit tests (K-S test). The K-S test was found suitable for the investigation as it can be used for continuous random variables and does not require the grouping of data.

\section{STATISTICAL INVESTIGATION OF THE CURRENT DATA}

Summaries of the probabilistic characteristics of the YS for NSHSS plates, $R_{e, \text { rule }}=235 \mathrm{MPa}$, with a strength level of $235, t \leq 50 \mathrm{~mm}$, are provided in this section. These characteristics include, inter alia, the arithmetic mean, the median, the standard deviation, the coefficient of variation, and the underlying probability distribution for YS.

The sample tested in this investigation included 2,198 plates of a thickness of up to $50 \mathrm{~mm}$ manufactured at four steelworks in 1990 and 1991. Fig. 1. presents the sample (empirical) histogram of plate thickness. A cursory look at the set allows for the conclusion that it was dominated by plates of a thickness of between 5 and 25 millimeters (approx. 90\% of the sample). The average value of plate thickness in the sample was $14.0 \mathrm{~mm}$, the most frequently value was 10.0 $\mathrm{mm}$, and the median was $12.0 \mathrm{~mm}$.

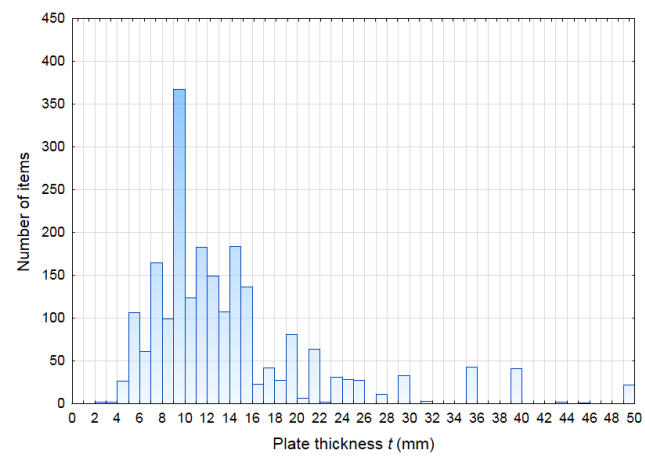

Fig.1. Sample histogram of plate thickness

Fig. 2. presents the empirical distribution of specific plate YS frequency for the sample. Roughly speaking, it can be assessed that 
most of the plates had a YS ranging from 270 to $350 \mathrm{MPa}$ (approx. $90 \%$ of the sample).

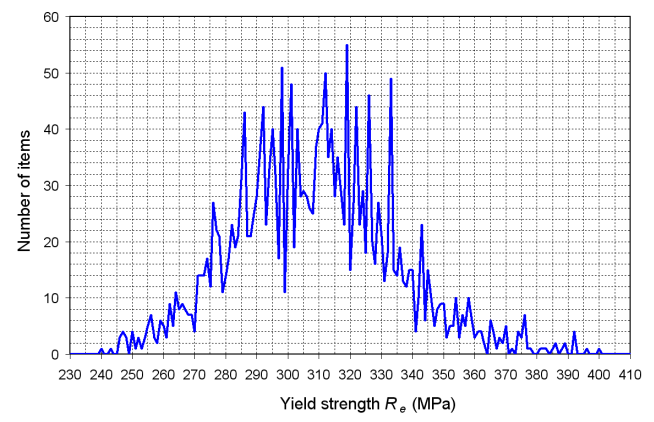

Fig.2. Sample (empirical) frequency distribution of yield strength

Table 1. presents the statistical parameters for the sample. In particular, it was found that the mean value of the YS for the sample was $308.3 \mathrm{MPa}$, and the median was 308.0 $\mathrm{MPa}$, while the most frequent value was 319.0 MPa. The coefficient of variation $(\mathrm{COV})$ was $8.19 \%$. The standard deviation $(S D V)$ was $25.24 \mathrm{MPa} .25 \%$ of the plates had a YS within the range from 240.0 MPa to 291.0 MPa, and another $25 \%$ of the plates were within the range from $324.0 \mathrm{MPa}$ to $400.0 \mathrm{MPa}$. The remaining $50 \%$ of the plates were within the YS range from 291.0 $\mathrm{MPa}$ to 324.0 MPa.

Table 1 also shows that for the sample tested, the (average) difference bias $b_{D}=73.3$ $\mathrm{MPa}$ (average excess yield strength); the (average) ratio bias was $b_{R}=1.312$.

As the data sample of the YS values was drawn from a population of values (plates), inferences about the population were drawn from certain sample statistics and assumptions about the manner in which the population of values was distributed. A sample histogram (see Fig. 4.) can aid in our attempt to picture the population distribution. The normal distribution is ordinarily assumed to be the underlying distribution for repeatability or random error. Hence, the statistical analysis of samples is often based on the assumption that the data follow the normal distribu- tion. Consequently, it is often necessary to assess whether the data are indeed normally distributed or a least approximately normally distributed NASA (2010).

Table.1. Statistical data on the yield strength.

\begin{tabular}{|l|r|l|}
\hline \multicolumn{3}{|l|}{ Measures of statistical locations } \\
\hline sample size: & 2198 & - \\
\hline arithmetic mean: & 308.3 & $\mathrm{MPa}$ \\
\hline median: & 308.0 & $\mathrm{MPa}$ \\
\hline mode: & 319.0 & $\mathrm{MPa}$ \\
\hline
\end{tabular}

Measures of statistical dispersion; moments

\begin{tabular}{|l|r|l|}
\hline standard deviation: & 25.24 & $\mathrm{MPa}$ \\
\hline coefficient of variation: & 8.19 & $\%$ \\
\hline variance: & 637.28 & $\mathrm{MPa}^{2}$ \\
\hline Uncertainty measures & \multicolumn{2}{|l|}{} \\
\hline (average) difference bias: & 73.3 & $\mathrm{MPa}$ \\
\hline (average) ratio bias: & 1.312 & - \\
\hline
\end{tabular}

As the data sample of the YS values was drawn from a population of values (plates), inferences about the population were drawn from certain sample statistics and assumptions about the manner in which the population of values was distributed. A sample histogram (see Fig. 3.) can aid in our attempt to picture the population distribution. The normal distribution is ordinarily assumed to be the underlying distribution for repeatability or random error. Hence, the statistical analysis of samples is often based on the assumption that the data follow the normal distribution. Consequently, it is often necessary to assess whether the data are indeed normally distributed or a least approximately normally distributed NASA (2010).

Both qualitative and quantitative methods can be used to determine if the sampled data can be assumed to be normally distributed. Qualitative or graphical methods include the use of a frequency histogram a normal probability plots. Quantitative or sta- 
tistical methods include the 68-95-99.7 rule, the Kolmogorov-Smirnov test, and variations of these tests. While graphical techniques provide a visual depiction of the data, their interpretation can be highly subjective. Statistical tests provide more formal, objective methods for assessing whether the normal distribution provides an adequate description of the observed data.

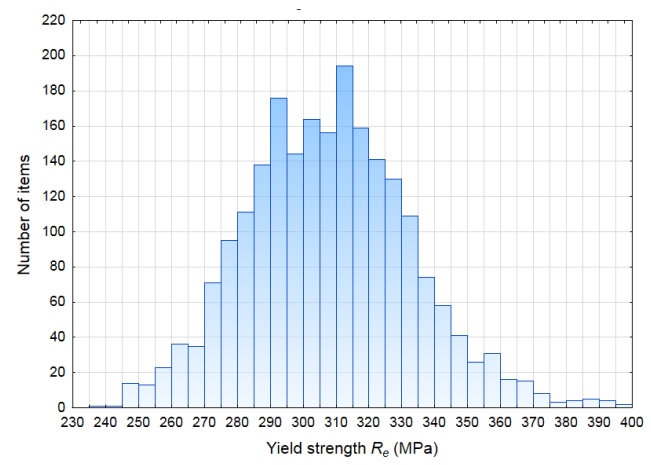

Fig.3. Sample (empirical) histogram of yield strength

The 68-95-99.7 rule may be used to quickly obtain a rough determination of whether or not the YS distribution is normal. For normally distributed data, approx. $68 \%$ of such data should be expected to lie more than 1 standard deviation below the mean and above the mean (i.e., within the range of $[308.3-25.24=283.06 \mathrm{MPa}, 308.3+25.24$ $=333.54 \mathrm{MPa}])-$ in fact that is $69.17 \%$. Also, approx. $13 \%$ of such data should be expected to lie within the range from 333.54 $\mathrm{MPa}$ to $358.78 \mathrm{MPa}$ - in fact that is $13.81 \%$. Also, approx. $13 \%$ of such data should be expected to lie within the range from 257.82 $\mathrm{MPa}$ to $283.06 \mathrm{MPa}$ - in fact that is $13.94 \%$. This shows that the actual distribution of YS in a population might be normal, see also Fig. 4. However, more precisely, quantitative method is needed a way to look at how the data is distributed. Such a method will be described and used in the following.

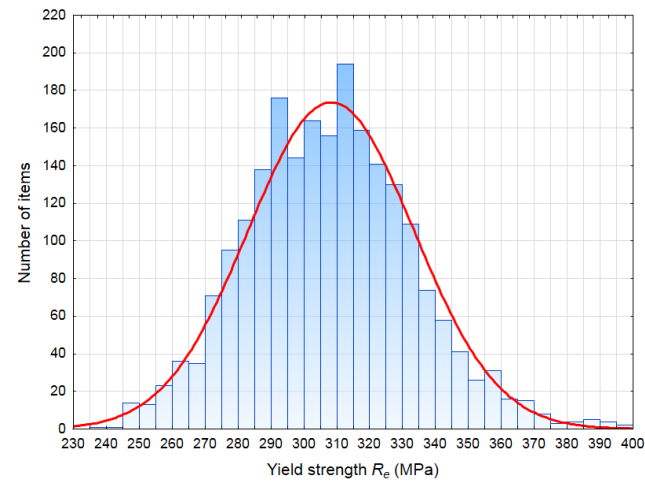

Fig.4. A sample histogram of yield strength and a normal probability distribution curve

In order to enable the collected data to be used for simulation calculations, a mathematical model of probability distribution of yield strength had been proposed. The model would allow for the sampling of plate yield strength values concordant with a specific probability distribution, and would include (1) the distribution type, and (2) the values of the parameters of that distribution. This could be, for instance, (1) a normal distribution, and (2) the mean value and the standard deviation. Based on the author's former experience, three theoretical probability distributions were considered: the normal distribution, the lognormal distribution and the Weibull distribution.

The Kolmogorov-Smirnov goodness-offit test (or shortly: the K-S test) was used for verifying whether or not the proposed theoretical probability distribution could represent an actual distribution of yield strength in a population. The test is defined by: (1) $H_{0}$ : the data follow a specifc (assumed, theoretical) distribution (the null hypothesis), $H_{a}$ : the data do not follow a specific distribution (the alternative hypotesis); (2) test statistic: $d:=\max _{\mathrm{x}}|D(x)|=$ $\max _{x}\left|F_{n}(x)-F(x)\right|$, where $F(x)$ is the theoretical cumulative distribution of the distribution being tested, $F_{n}(x)$ is the empirical cumulative distribution; (3) significance level: $\alpha$; (4) critical values: the 
hypothesis regarding the distributional form is rejected if the test statistic, $d(d \mathrm{~K}-\mathrm{S})$, is greater than the critical value obtained from a respective table.

The K-S test provides adequate means of determining whether or not the data are sampled from a specific distribution.

Let us formulate the problem. In 1990 and 1991, data from Polish Register of Shipping certificates were collected regarding the actual YS values for NSHSS plates, with a strength level of 235 and a maximum thickness of $50 \mathrm{~mm}$, manufactured at four European steelworks. The gathered sample contained information on 2,198 plates. The Kolmogorov-Smirnov goodness-of-fit test, with a significance level $\alpha=0.05$, had to be used to verify three hypotheses claiming that in the general population of the plates, the YS demonstrated the following distributions: (1) normal $N(308.2589,25.2444)$, (2) lognormal $L N(5.7276,0.0817)$, or (3) Weibull $W(320.0636,12.1653)$. The numerical values of the distribution parameters corresponding to the best fit of the theoretical distribution to the empirical data, were obtained using the Dell $^{\text {TM }}$ Statistica ${ }^{\text {TM }} 13.1$ software.

The sample (empirical) cumulative distribution and theoretical cumulative distributions are presented on Fig. 5. The maximum value of the distances of the sample (empirical) cumulative distribution from the theoretical cumulative distributions, $D(x)$, is Kolmogorov-Smirnov statiscics, $d=\mathrm{K}-\mathrm{S}$, for each theoretical distribution.

Statistica $^{\mathrm{TM}}$ 13.1, an analytics software package by Dell ${ }^{\mathrm{TM}}$, was used for calculations made for the purpose of choosing the theoretical probability distribution (as a reminder: the distribution type and parameters) that was the best fit to the collected data. For the probability distribution types assumed by the user (in the paper, the normal, lognormal and Weibull distributions were assumed) the software automatically determined the values of distribution parameters that ensured the best fit to the data. In order to examine the fit quality, the author used the one sample Kolmogorov-Smirnov test (K-S test) procedure offered by the software, which tests whether the sample (empirical) distribution differs substantially from theoretical expectations.

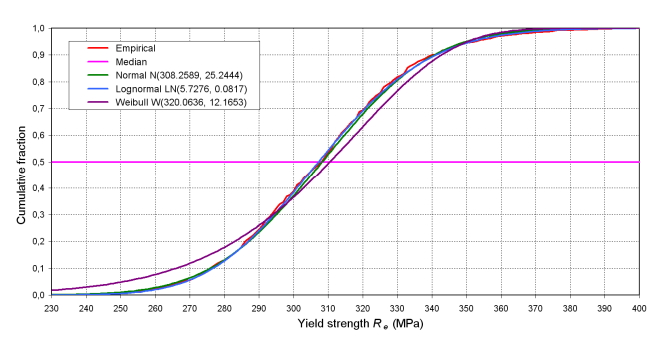

Fig.5. The cumulative fraction of the sample (empirical) probability distribution and theoretical probability distributions

The statistics that was examined in this test, $d \mathrm{~K}-\mathrm{S}$, was the largest distance, $D(x) \mathrm{K}$ $\mathrm{S}$, between the actual cumulative distribution function of the colelcted data and the cumulative distribution function of the theoretical distribution being examined with the determined parameter values. For each of the distributions, the parameter values that would minimize that distance were sought. Apart from the distribution parameter values, the software also calculated the value of the $p$ statistic that described the quality of the fit of the theoretical distribution.

How to interpret the $p$-value? In this paper, the collected data were compared to the preassumed theoretical probability distribution with the aim of determining whether the empirical data would produce a theoretical probability distribution. If both the probability distributions were to be "the same" (the null hypothesis), it was assumed that the sample (empirical) probability distribution would be a good approximation of the theoretical probability distribution. Rarely are the two probability distributions identical, which renders the question of how different these probability must be. Statistics aim to assign numbers to the test results, and 
$p$-values report if the probability distributions differ significantly. The null hypothesis should be rejected if the $p$-value is "small".

If the $p$-value is less than the chosen significance level $(\alpha)$, this suggests that the observed data is sufficiently inconsistent with the null hypothesis that the null hypothesis may be rejected. However, that does not prove the tested hypothesis is true. For typical analysis, using the standard $\alpha=0.05$ cutoff, the null hypothesis is rejected when $p<$ 0.05 and not rejected when $p>0.05$. The $p$ value does not, in itself, support reasoning about the probabilities of hypotheses, but is only a tool for deciding whether to reject the null hypothesis.

In short, a large $p$-value calculated from the data indicates that the null hypothesis should not be rejected. The lower the $p$ value, the more unlikely the outcome, and the stronger the evidence is against the null hypothesis. The null hypothesis should be rejected if the evidence is strongly against it (OpenStax College 2015).

For each theoretical (assumed, tested) distribution, the statistical tests adopted the following scheme: (1) the null hypothesis $H_{0}$ claiming that the yield strength in the general population of plates was described with the proposed theoretical probability distribution (as a reminder: the distribution type and parameter values) and the alternative hypothesis $H_{a}$ claiming that the yield strength was described with a theoretical probability distribution different from the proposed one were assumed; (2) the significance level $\alpha=$ 0.05 was assumed; (3) the value of the parameter $p \mathrm{~K}-\mathrm{S}$ was calculated; (4) on the basis of a comparison between the values of the significance level $\alpha$ and $p \mathrm{~K}-\mathrm{S}$, a decision was recommended to either adopt or reject the proposed theoretical probability distribution.

The K-S test helped to identify data with the normal distribution N(308.2589, $25.2444): d \mathrm{~K}-\mathrm{S}=0.0274,(p=0.061)>(\alpha=$ $0.05)$. The high statistical significance value of $p=0.061$ meant that the results observed in the sample were very probable if the null hypothesis was true. The outcome was statistically insignificant at $p=0.061$. The statistically insignificant outcome could be interpreted as meaning that there was a $6.1 \%$ probability of obtaining the observed results, or even results indicating a stronger fit / compliance of the distribution, assuming that the null hypothesis was true.

The K-S test helped to identify data with the lognormal distribution $L N(5.7276$, $0.0817): d \mathrm{~K}-\mathrm{S}=0.027,(p=0.078)>(\alpha=$ $0.050)$. The high statistical significance value of $p=0.078$ meant that the results observed in the sample were very probable if the null hypothesis was true. The result was statistically insignificant at $p=0.078$. The statistically insignificant outcome could be interpreted as meaning that there was a $7.8 \%$ probability of obtaining the observed results, or even results indicating a stronger fit / compliance of the distribution, assuming that the null hypothesis was true.

The K-S test indicated that these data were unlikely to be Weibull distributed $W(320.0636,12.1653): d \mathrm{~K}-\mathrm{S}=0.072,(p=$ $0.000)<(\alpha=0.050)$. The low statistical significance value of $p=0.000$ meant that the results observed in the sample were unlikely if the null hypothesis was true. A statistically significant result was obtained at $p=0.000$. The statistically significant outcome could be interpreted as meaning that there was a near zero probability of obtaining the observed results, or even results indicating a stronger fit / agreement of the distribution, assuming that the null hypothesis was true.

The results of the conducted Kolmogorov-Smirnov tests allowed for the following conclusions to be drawn (see Fig. 6.):

1. with a likelihood of error of less than $5 \%$, it could be determined that the collected statistical data did not provide grounds for rejecting the theoretical normal $N(308.2589 ; 25.2444)$ and lognormal $L N(5.7276 ; \quad 0.0817) \quad$ distributions; therefore, both the proposed theoretical 
distributions could be assumed as correctly representing the general population of NSHSS plates of a thickness of up to 50 $\mathrm{mm}$;

2. on the basis of the collected data, the theoretical Weibull distribution $W(320.0636$; 12.1653) should be rejected; this distributions should not be assumed as correctly representing the general population of NSHSS plates of a thickness of up to 50 $\mathrm{mm}$.

Summarizing the above statements and the formulation based on the collected empirical data and calculations carried out with the use of the Dell ${ }^{\mathrm{TM}}$ Statistica $^{\mathrm{TM}} 13.1$ software, it can be concluded that the theoretical normal distribution $N(308.2589,25.2444)$ and the lognormal distribution $L N(5.7276$, 0.0817) corresponded to the collected empirical data. The theoretical Weibull distribution $W(320.0636,12.1653)$, as well as other distributions of this type, were to be rejected as significantly different from the empirical distribution.

As there were no grounds for rejecting the normal distribution representing the YS in the sample (which is shown above), the assumptions concerning the normality of distribution made and utilized earlier in this paper were accurate.

\section{CONCLUSIONS}

The material property that this investigation focused on was yield strength. Statistical estimates of the uncertainty associated with the material yield strength of normal strength hull structural steel plates with a thickness of up to $50 \mathrm{~mm}$ were presented. The yield strength data used were compiled from raw material measurements (before the material was used for ship construction).

The shape analysis showed that the sample (empirical) yield strength distribution of normal strength hull steel plates with a thickness of up to $50 \mathrm{~mm}$ was roughly symmetric; the sample (empirical) histogram suggested normality.
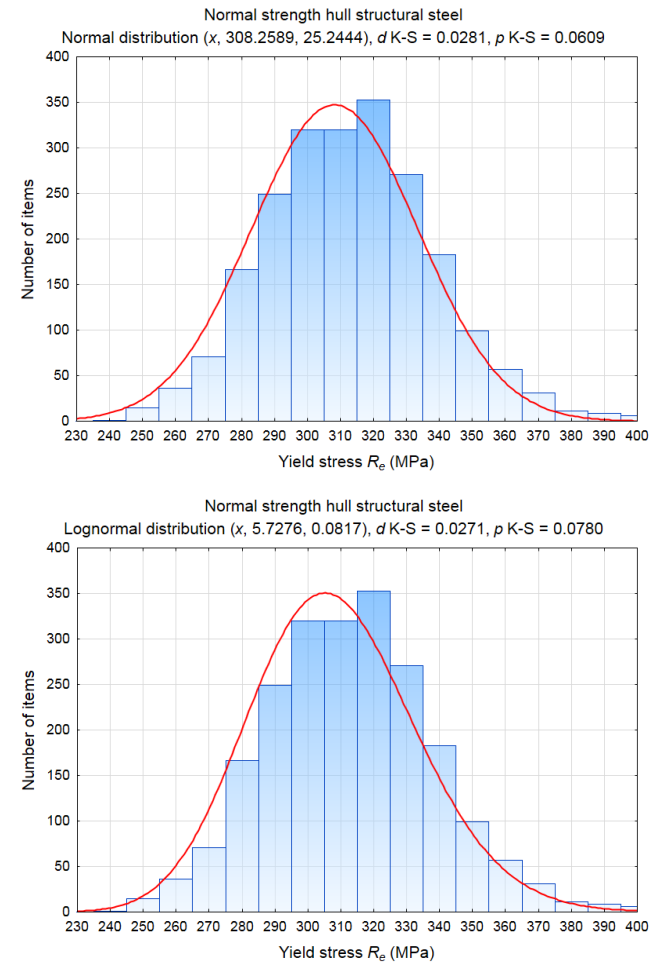

Normal strength hull structural stee

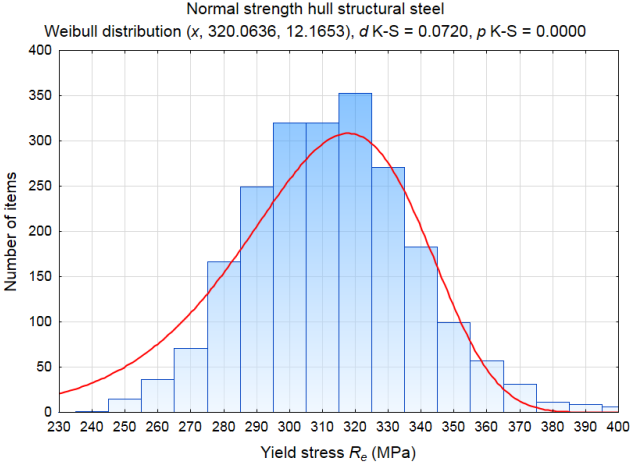

Fig.6. The theoretical probability distributions investigated of the yield strength of normal strength hull structural steel plates

The large values of the mean, 308.3 $\mathrm{MPa}$, the standard deviation, 25.24 $\mathrm{MPa}$, and the coefficient of variation, 0.082 , were influenced by the large yield strength ratio and variation of the data. The goodness-of-fit tests suggested that both the lognormal $L N(5.7276,0.0817)$ and normal 
$N(308.2589$, 25.2444) probability distributions with their accompanying statistics could be almost equally valid choices for describing the yield strength of normal strength hull structural steel plates.

The results provided in this paper can be used in reliability-based design and assessment when dealing with ship structural elements manufactured from such material nowadays, as well. However, caution should be exercised when using these results, since they might be revised as new data and research on the subject emerge.

\section{REFERENCES}

[1]. Collette, M. (2017) Uncertainty Approaches in Ship Structural Performance, R. Ghanem et al. (eds.), Handbook of Uncertainty Quantification, Springer International Publishing Switzerland, DOI 10.1007/978-3-31912385-1_48.

[2]. Hess, P.E., Ayyub, B.M (1997) Variability in Geometry and Imperfections of Surface Ship Structural Scantlings, Technical report, NSWCCD-TR-65-97/02, Naval Surface Warfare Center, Carderock Division, West Bethesda.
[3]. Hess, P.E., Bruchman, D., Assakkaf, I.A., Ayyub, B.M. (2002) Uncertainties in Material and Geometric Strength and Load Variables, Naval Engineers Journal, 114(2): 139 $-166$.

[4]. IACS (2017) UR W11 Normal and higher strength hull structural steels, Rev. 9 May 2017.

[5]. NASA (2010) NASA HANDBOOK: Measurement Uncertainty Analysis Principles and Methods. NASA Measurement Quality Assurance Handbook - ANNEX 3, NASAHDBK-8739.19-3, National Aeronautics and Space Administration, Washington DC 20546.

[6]. OpenStax College (2015) Introductory Statistics, University of Oklahoma Custom Edition, Rice University, Houston, Texas, USA.

[7]. Simiu, E. and Smith, Ch.E. (1984) Structural Reliability Fundamentals and Their Application to Offshore Structures, Minerals Management Service, U.S. Department of the Interior, Reston, VA 22091. September 1984.

Paper received on November $6^{\text {th }}, 2019$ 
